\title{
Low-frequency pulsed electromagnetic field promotes functional recovery, reduces inflammation and oxidative stress, and enhances HSP70 expression following spinal cord injury
}

\author{
CHUNYAN WANG $^{1 *}$, YANG LIU ${ }^{2 *}$, YAO WANG $^{2}$, ZHIJIAN WEI $^{2}$, DONGMEI SUO $^{1}$, \\ GUANGZHI NING ${ }^{2}$, QIULI WU ${ }^{2}$, SHIQING FENG ${ }^{2}$ and CHUNXIAO WAN $^{1}$ \\ Departments of ${ }^{1}$ Rehabilitation Medicine and ${ }^{2}$ Orthopedics, \\ Tianjin Medical University General Hospital, Tianjin 300052, P.R. China
}

Received April 20,2018; Accepted November 23, 2018

DOI: $10.3892 / \mathrm{mmr} .2019 .9820$

\begin{abstract}
Low-frequency pulsed electromagnetic fields (LPEMFs) have been reported to be protective for multiple diseases. However, whether the administration of LPEMFs inhibits inflammation and oxidative stress following spinal cord injury requires further investigation. In the current study, a contusion spinal cord injury model was used and LPEMFs administration was applied to investigate the molecular changes, including inflammation, oxidative stress and heat shock protein 70 (HSP70) levels. The results revealed that LPEMFs significantly promoted functional recovery following spinal cord injury, as demonstrated by an increased Basso, Beattie and Bresnahan score. The results demonstrated that LPEMFs decreased the expression of inflammatory factors, including tumor necrosis factor- $\alpha$, interleukin- $1 \beta$ and nuclear factor- $\mathrm{kB}$. Additionally, LPEMFs exposure reduced the levels of inducible nitric oxide synthase and reactive oxygen species, and upregulated the expression of catalase and superoxide dismutase. Furthermore, treatment with LPEMFs significantly enhanced the expression of HSP70 in spinal cord-injured rats. Overall, the present study revealed that LPEMFs promote functional recovery following spinal cord injury, potentially by modulating inflammation, oxidative stress and HSP70.
\end{abstract}

Correspondence to: Professor Shiqing Feng, Department of Orthopedics, Tianjin Medical University General Hospital, 154 Anshan Road, Heping, Tianjin 300052, P.R. China

E-mail: sqfeng@tmu.edu.cn

Professor Chunxiao Wan, Department of Rehabilitation Medicine, Tianjin Medical University General Hospital, 154 Anshan Road, Heping, Tianjin 300052, P.R. China

E-mail:wcx2226@163.com

*Contributed equally

Key words: low-frequency pulsed electromagnetic fields, spinal cord injury, inflammation, oxidative stress, heat shock protein 70

\section{Introduction}

Spinal cord injury (SCI) causes severe damage to the central nervous system, resulting in irreversible motor and sensory dysfunction below the injury area (1). In modern society, the global incidence of SCI varied from 8 to 246 per million depending on the country or region (2), and an increasing number of paralyzed patients survive longer, experiencing a variety of complications $(3,4)$. Currently there are no effective therapeutic treatments for SCI. Methylprednisolone, which was previously used as first-aid measure for acute SCI, is no longer recommended in the guidelines of AANS/CNS as of 2013 due to an increased occurrence of complications and no strong evidence of clinical efficacy (5). Rehabilitation, the only proven effective treatment for SCI, is limited for early application as it is only suitable for less serious injuries and requires active cooperation from patients (6). Thus, novel noninvasive treatments are required as early interventions for patients with SCI.

The pathological process of SCI can be divided into two stages; the primary and secondary injury (7). The primary injury is the original tissue breakdown caused by contusion or compression, which occurs immediately after SCI. The secondary injury is a series of pathologic changes following the primary injury, including an increase in the permeability of the blood spinal cord barrier, the infiltration of inflammatory cells, excitotoxicity, demyelination and neuronal apoptosis, which lasts for days to months. Two of the most important components of the secondary injury in its early stages are inflammation and oxidative stress. The inflammation includes increased expression of pro-inflammatory factors, including tumor necrosis factor- $\alpha$ (TNF- $\alpha$ ) and interleukin-1 $\beta$ (IL-1 $1 \beta$ ), and a reduction in inflammatory mediators (8). The oxidative stress involves an increase in the levels of nitric oxide (NO) and reactive oxygen species (ROS) (9).

The use of low-frequency pulsed electromagnetic fields (LPEMFs) is a noninvasive therapeutic method for various diseases. Recent evidence has demonstrated that LPEMFs can prevent inflammation and oxidative stress. LPEMF stimulation can suppress the production of IL- $1 \beta$ and TNF- $\alpha$ in cultured nucleus pulposus cells (10). Furthermore, LPEMFs can reduce 
ROS levels and enhance antioxidative stress responses in osteoblasts (11). LPEMFs exhibit strong neuroprotective effects in the nervous system. In ischemic stroke, LPEMFs can promote functional recovery by activation of the brain derived neurotrophic factor/tropomyosin receptor kinase B/protein kinase B signaling pathway (12). Additionally, LPEMFs can modulate the expression of microRNAs and stimulate tissue regeneration in in vitro models of Alzheimer's disease (13). Recent studies have revealed that extremely low-frequency magnetic fields reduce iron-induced tissue damage following SCI (14). However, whether the administration of LPEMFs inhibits the early-stage reaction of SCI secondary injury, inflammation and oxidative stress, requires further exploration. In the present study, neuroprotective effects of LPEMF stimulation on SCI model rats were investigated. The changes in inflammation and oxidative stress molecular markers were then compared, and whether this protective effect was modulated by targeting heat shock protein 70 (HSP70) was examined. The results of the current study may provide a noninvasive alternative therapeutic method for the early treatment of SCI.

\section{Materials and methods}

Experimental animals. Adult female Wistar rats $(230 \pm 20 \mathrm{~g}$; $\mathrm{n}=60$ ) used in the current study were all provided by Tianjin Medical University Animal Research Center (Tianjin, China; permission no. SCXK-2012-0004). All animal experiments were approved by the Animal Welfare Committee of Tianjin Medical University (Tianjin, China), which is based on the NIH Guide for the Care and Use of Laboratory Animals (15). The rats were randomly distributed into three groups: Sham group, SCI group and LPEMF group. In the sham group, rats underwent laminectomy, and the spinal cord was not injured. In the SCI group, the spinal cord was injured using the same procedure used in the LPEMF group on an identical electromagnetic device, but without the application of LPEMFs. In the LPEMF group, Wistar rats received the LPEMFs treatment for $1 \mathrm{~h}$ per day from $24 \mathrm{~h}$ after SCI. Animals were sacrificed on days 3, 7 and 14 after SCI, and the spinal cords were harvested for further analysis.

Contusion SCI model. The standard New York University impactor machine was used to induce a spinal cord contusion injury model as described previously (16). Rats were anesthetized with chloral hydrate $(300 \mathrm{mg} / \mathrm{kg})$, and a laminectomy was performed to expose the T10 spinal cord. A metal rod (10 g, $25 \mathrm{~mm}$ ) was dropped onto the back side of the spinal cord. A surveillance system was used to control the compression force and velocity to maintain uniformity between animals. Following the operation, the bladders of these rats were manually emptied.

LPEMFs treatment. The BG100A-2 pulsed magnet field therapeutic apparatus (Concord Beijing Medical Equipment Co., Ltd., Beijing, China; patent no. ZL00101667.9) was used in the current study. The apparatus contains seven coils arranged end-to-end beneath the treatment table and a magnetic line of force was positioned across the rats longitudinally. The frequency, power and duty cycle are all adjustable and the apparatus can be monitored and controlled by computer system. In the LPEMFs group, rats were exposed to LPEMFs (frequency, $50 \mathrm{~Hz}$; power, $2.5 \mathrm{mT}$; duty cycle, 40\%) at $24 \mathrm{~h}$ after SCI. Rats were placed into a transparent plastic chamber with ventilation and were given time to explore for $1 \mathrm{~h}$ per day (8:00-9:00 a.m.) for 14 days. In the SCI group, animals were placed in the same chambers on the treatment table for $1 \mathrm{~h}$ per day without exposure to LPEMFs.

Assessment of locomotor activity. Functional recovery of the animals was evaluated using the Basso Beattie Bresnahan (BBB) locomotor rating scale (17). The BBB was observed by two groups, and the observers were blinded to the design of current experiment (18). The BBB scores of rats were recorded prior to contusion operation and at 1,3, 5, 7 and 14 days post-injury (dpi).

Enzyme-linked immunosorbent assay (ELISA). The spinal cord tissue (10 mm block of spinal cord surrounding the lesion center) was collected and homogenized at $14 \mathrm{dpi}$. Then, the tissue homogenate was centrifuged at a speed of $10,000 \mathrm{x} \mathrm{g}$ at $4^{\circ} \mathrm{C}$ for $20 \mathrm{~min}$ and the supernatant was collected for determining protein concentration using a Pierce ${ }^{\mathrm{TM}}$ BCA Protein Assay Kit (Thermo Fisher Scientific Inc., Waltham, MA, USA). The commercially available ELISA kits were obtained from the following companies: TNF- $\alpha$ ELISA kit (cat. no. ab46070; Abcam, Cambridge, UK), IL-1 $\beta$ ELISA kit (cat. no. RA20422; Bio-Swamp, Wuhan, China), superoxide dismutase (SOD) ELISA kit (cat. no. 706002; Cayman Chemical Company, Ann Arbor, MI, USA), catalase (CAT) ELISA kit (cat. no. 11363727001; Sigma-Aldrich; Merck KGaA, Darmstadt, Germany).

Western blot analysis. After 14 days of treatment or SCI, the spinal cord tissue around the lesion center was collected, harvested and homogenized in radioimmunoprecipitation assay lysis buffer (cat. no. P0013B; Beyotime Institute of Biotechnology, Shanghai, China). The concentration of protein was detected using bicinchoninic acid protein assay kit (Thermo Fisher Scientific, Inc.). Equal amounts of protein samples $(50 \mu \mathrm{g})$ from three individual animals in each group were resolved using SDS-PAGE (12\% gel) for separation and then transferred to a polyvinylidene difluoride membrane (EMD Millipore, Billerica, MA, USA). The membrane was blocked with $5 \%$ non-fat milk and incubated with anti-inducible nitric oxide synthase (iNOS; 1:250; cat. no. ab15323; Abcam) and anti- $\beta$-actin (1:2,000; cat. no. ab8227; Abcam) overnight at $4^{\circ} \mathrm{C}$. Then, the membrane was incubated with secondary horseradish peroxidase (HRP)-conjugated goat anti-rabbit antibody (1:10,000; cat. no. ab205718; Abcam) at $37^{\circ} \mathrm{C}$ for $1 \mathrm{~h}$. BeyoECL Star (cat. no. P0018AM; Beyotime Institute of Biotechnology) was used to develop the HRP signal. Signals were captured using a ChemiDoc MP System (Bio-Rad Laboratories, Inc., Hercules, CA, USA) and quantified using ImageJ software version 1.32 (National Institutes of Health, Bethesda, MD, USA). The expression levels of iNOS were determined following normalization to $\beta$-actin levels.

Immunohistochemistry analysis. Spinal cords were collected 14 days after injury and samples were quickly frozen at $-40^{\circ} \mathrm{C}$ immersed in $4 \%$ paraformaldehyde. Transverse 
$10 \mu \mathrm{m}$ thick sections of spinal cord were used for immunohistochemistry analysis. Following permeabilization in $0.25 \%$ Triton $\mathrm{X}-100 / \mathrm{PBS}$ for $10 \mathrm{~min}$ at room temperature, sections were blocked with $10 \%$ goat serum (cat. no. SL038; Beijing Solarbio Science \& Technology Co., Ltd., Beijing, China) for $60 \mathrm{~min}$ at room temperature. Sections were stained using anti-nuclear factor- $\kappa \mathrm{B}(\mathrm{NF}-\kappa \mathrm{B})$ p65 (1:2,000; cat. no. ab16502; Abcam) and anti-HSP70 (1:100; ab79852; Abcam) at $4^{\circ} \mathrm{C}$ overnight. Samples were incubated with secondary HRP-conjugated goat anti-rabbit antibody (1:10,000; cat. no. ab205718; Abcam) at $37^{\circ} \mathrm{C}$ for $1 \mathrm{~h}$. The DAB Horseradish Peroxidase Color Development kit (cat. no. P0202; Beyotime Institute of Biotechnology) was used for signal development (sections were incubated at room temperature for $25 \mathrm{~min}$ ). Then, 3 fields were randomly selected for each sample, and the positive area in each field was collected and quantified using ImageJ software version 1.32 (National Institutes of Health).

ROS assay. The spinal cord tissue (5 mm block of spinal cord surrounding the lesion center) was collected and homogenized at 14 dpi. Subsequently, the tissue homogenate was centrifuged $\left(1,500 \mathrm{x} \mathrm{g}\right.$ at $4^{\circ} \mathrm{C}$ for $\left.20 \mathrm{~min}\right)$, and the supernatant was collected. A ROS assay kit (cat. no. D6883; Sigma-Aldrich; Merck $\mathrm{KGaA}$ ) was used to determine the production of ROS. The supernatant was incubated with 2,7-dichlorodihydrofluorescein diacetate for $1 \mathrm{~h}$ and then washed twice with PBS in ice. Fluorescence levels were detected at 480/530 nm.

Statistical analysis. The experimental data are expressed as the mean \pm standard error. A one-way analysis of variance followed by Tukey's post hoc tests for multiple comparisons were used to analyze data (SPSS 19.0 software; IBM Corp., Armonk, NY, USA). P<0.05 were considered to indicate a statistically significant difference.

\section{Results}

Protective effects of LPEMFs on locomotor recovery following SCI in rats. To evaluate whether the LPEMF treatment has protective effects on motor function in SCI rats, the BBB locomotor rating scale was used to measure behavior for 2 weeks. As presented in Fig. 1, all the animals exhibited full marks (21 points) in $\mathrm{BBB}$ scoring prior to the injury. At dpi 1, the BBB scores dropped to 0 . Following SCI, rats exhibited spontaneous functional recovery over time and there were no significant differences between the SCI group and the LPEMF group before dpi 7. However, the rats exhibited better motor function recovery after 7 dpi under LPEMF treatment compared with SCI rats, and the BBB scores were significantly different ay dpi 7 and $14(\mathrm{P}<0.05)$. These results suggested that LPEMF exposure can promote locomotor recovery in SCI rats (Fig. 1).

Protective effect of LPEMFs on expression of pro-inflammatory cytokines in SCI rats. To determine whether LPEMFs suppressed inflammatory reaction by decreasing the secretion of pro-inflammatory cytokines in the injured spinal cord, the expression levels of TNF- $\alpha$ and IL-1 $\beta$ were assessed. Following SCI, the inflammation markers TNF- $\alpha$ and IL-1 $\beta$ were significantly increased compared with the Sham group (Fig. 2A and B). However, after 2 weeks of LPEMF

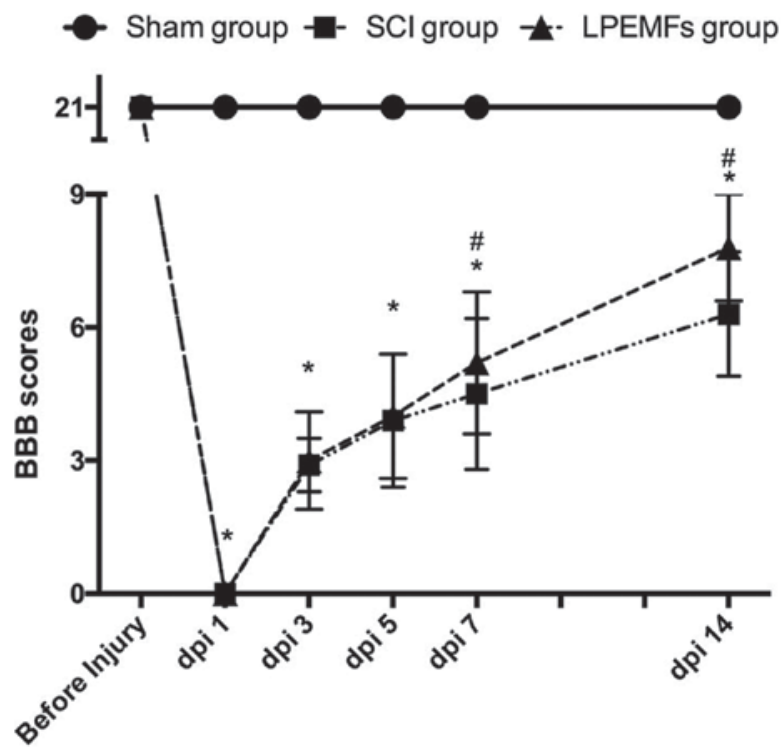

Figure 1. BBB scores in Sham group, SCI group and LPEMF group at different time points. ${ }^{*} \mathrm{P}<0.01$ vs. Sham group; ${ }^{\#} \mathrm{P}<0.05$ vs. SCI group. BBB, Basso Beattie Bresnahan; SCI, spinal cord injury; LPEMF, low-frequency pulsed electromagnetic fields.

treatment, the expression of TNF- $\alpha$ and IL-1 $\beta$ were decreased in comparison with the SCI group (Fig. 2A and B). These results indicated that LPEMF treatment, to a certain extent, can alleviate the inflammatory reaction following SCI.

Suppressive effect of LPEMFs on $N F-\kappa B$ expression in injured spinal cord. $\mathrm{NF}-\kappa \mathrm{B}$ is an important transcription factor that stimulates inflammation (19). To determine whether LPEMF treatment could suppress the expression of $N F-\kappa B$ in the injured spinal cord, particularly in the ventral horn, $\mathrm{NF}-\kappa \mathrm{B}$ protein levels were evaluated using immunohistochemistry (Fig. 3A). In the sham group, the $\mathrm{NF}-\kappa \mathrm{B}$ was difficult to detect (Fig. 3B). However, after 2 weeks of injury, there was a strong, positive $\mathrm{NF}-\kappa \mathrm{B}$ signal in the ventral horn of the spinal cord, which contains motor neurons (Fig. 3C). By contrast, the administration of LPEMFs significantly reduced the immunoreactivity of $\mathrm{NF}-\kappa \mathrm{B}$ in SCI rats (Fig. 3D). The quantified result was consistent with observation of sections (Fig. 3E).

Suppressive effect of LPEMFs on iNOS expression in injured spinal cord. To determine the iNOS expression following SCI, the iNOS was detected using western blot analysis. Significantly higher levels of the iNOS protein were detected following SCI compared with the Sham group. By contrast, the LPEMF treatment suppressed the iNOS protein expression in the injured spinal cord (Fig. 4A and B). The decreased iNOS expression indicated that the LPEMFs can alleviate oxidative stress following SCI.

Protective effect of LPEMFs on ROS production in SCI rats. To investigate the protective effect of LPEMFs on ROS production in SCI rats, the ROS levels between groups were measured. As demonstrated Fig. 5, the injury induced strong ROS production in spinal cord tissue compared with those in the uninjured Sham group. However, the LPEMF administration reduced the ROS level significantly compared with 

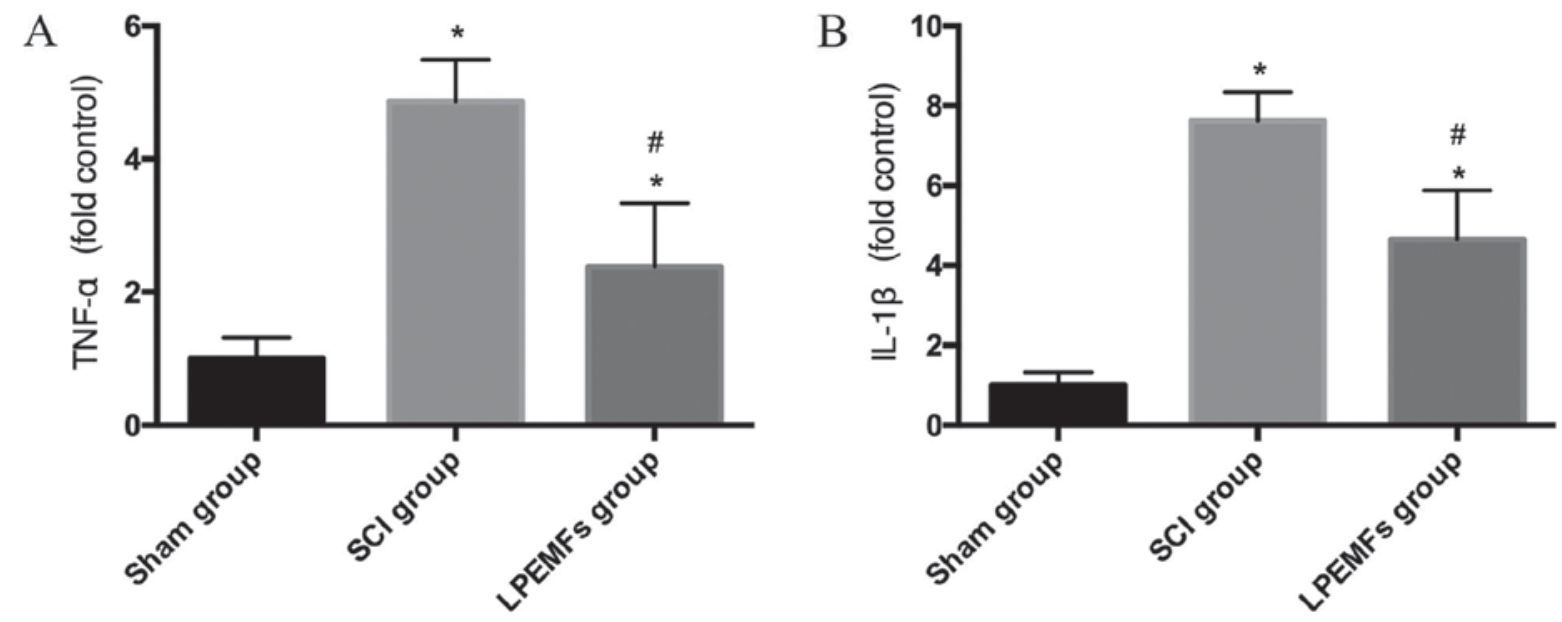

Figure 2. ELISA analysis of expression levels of TNF- $\alpha$ and IL-1 $\beta$ in the spinal cord. The ELISA was conducted at 14 days post-injury. (A) Expression levels of TNF- $\alpha$. (B) Expression levels of IL-1 $\beta$. " $\mathrm{P}<0.01$ vs. Sham group; ${ }^{\# \mathrm{P}}<0.05$ vs. SCI group. TNF- $\alpha$, tumor necrosis factor- $\alpha$; IL- $1 \beta$, interleukin- $1 \beta$; SCI, spinal cord injury; LPEMF, low-frequency pulsed electromagnetic fields.

SCI rats. This result indicated that LPEMFs can alleviate the oxidative stress by reducing ROS production following SCI.

Protective effect of LPEMFs on expression of antioxidant enzymes following SCI. To explore whether LPEMFs can alleviate oxidative stress through upregulation of antioxidant enzymes, the expression of SOD and CAT in spinal cord was measured using ELISA. Compared with the intact spinal cord, the injured tissue exhibited decreased expression of SOD and CAT. By contrast, the treatment of LPEMFs can reversed this reduction to a certain extent (Fig. 6A and B). These results provided evidence that the protective effect of LPEMFs on oxidative stress may be attributed to the upregulation of antioxidant enzymes.

Protective effect of LPEMFs on expression of HSP70 SCI rats. HSP70 is deemed as the protective agent for inflammation and oxidative stress during tissue damage. In the current study, the expression of HSP70 in the spinal cord following injury with and without the administration of LPEMFs was examined. Following SCI, the expression of HSP70 became scattered in the ventral horn compared with the spinal cord in the Sham group. However, the LPEMF treatment significantly increased the expression of HSP70 in motor neurons (Fig. 7A-D). The quantified result was consistent with observations (Fig. 7E). These results indicated that the anti-inflammatory and antioxidative stress effects of LPEMFs may associated with the high expression of HSP70.

\section{Discussion}

Electromagnetic fields (EMFs) have long been deemed relevant for human health (20), and recent studies have indicated that LPEMFs exhibit protective effects in multiple pathologies, including Alzheimer's disease (13), stroke (12), wound healing (21) and pain (22). The definition of low frequency is $<300 \mathrm{~Hz}$ and research has demonstrated that pulsed EMFs exhibited greater therapeutic effects when applied with an amplitude $<3 \mathrm{mT}$ and frequencies $<100 \mathrm{~Hz}$ (23). In the current study, LPEMFs with a $50 \mathrm{~Hz}$ frequency and $2.5 \mathrm{mT}$
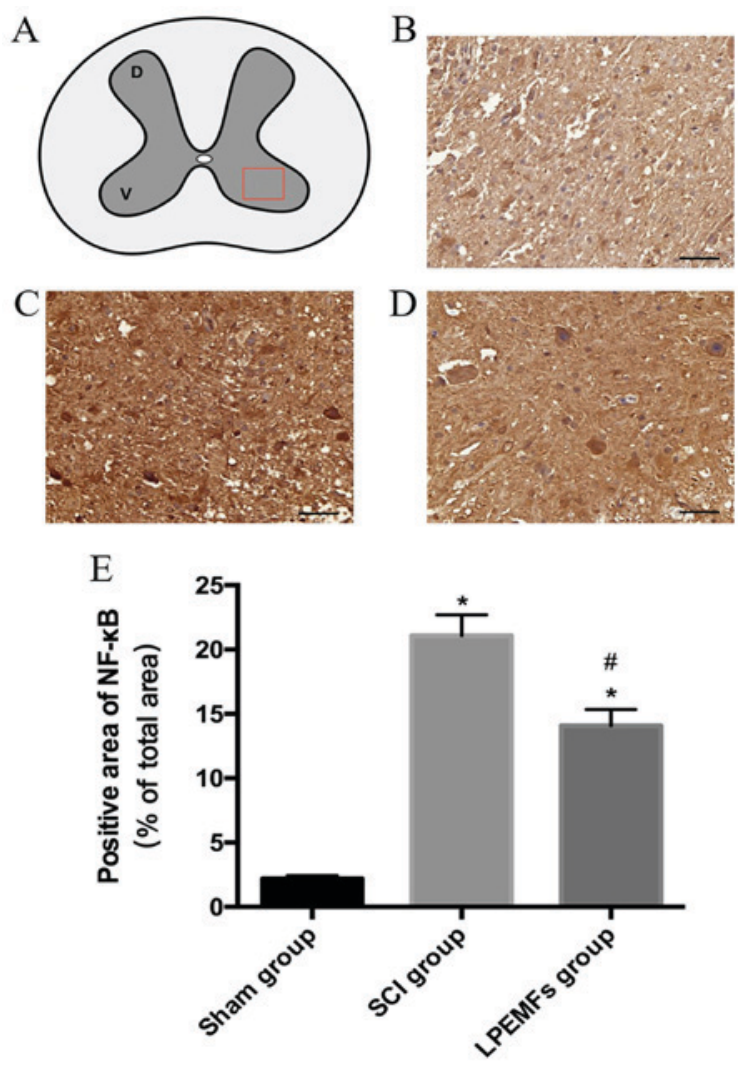

Figure 3. Expression of $\mathrm{NF}-\kappa \mathrm{B}$ in the ventral horn of the spinal cord. The NF- $\kappa \mathrm{B}$ was detected at 14 days post-injury. (A) Diagram of the spinal cord. Representative image of $\mathrm{NF}-\kappa \mathrm{B}$ expression in the ventral horn of the spinal cord in the (B) sham group, (C) SCI group and (D) LPEMF group. Scale bar, $50 \mu \mathrm{m}$. (E) Statistical comparison among the three groups. ${ }^{*} \mathrm{P}<0.01 \mathrm{vs}$. Sham group; ${ }^{\text {P }}<0.05$ vs. SCI group. NF- $\kappa \mathrm{B}$, nuclear factor- $\kappa \mathrm{B}$; SCI, spinal cord injury; LPEMF, low-frequency pulsed electromagnetic fields.

amplitude were applied, which are suitable parameters for the exploratory research. However, to the best of our knowledge, the current study was first administration of LPEMFs in a contusion SCI model, which is more clinically relevant than the transection model $(24,25)$. Unlike other disorders, the pathological changes following SCI are complex due to 

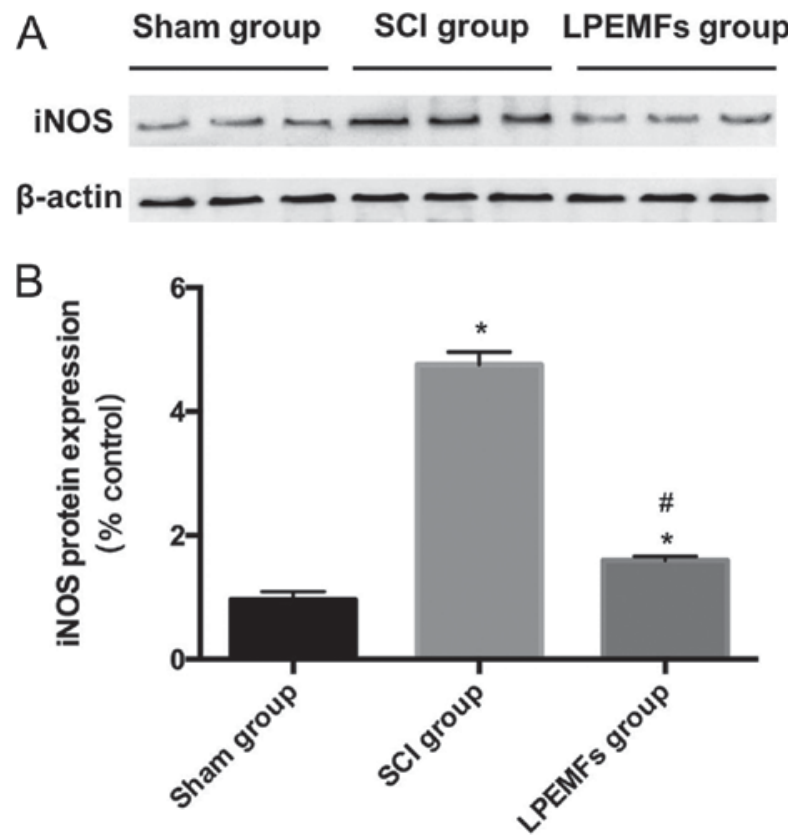

Figure 4. Effect of LPEMFs on the iNOS expression following SCI. The iNOS was detected at 14 days post-injury. (A) Western blot and (B) quantification was used to detect the expression of iNOS in sham, SCI and LPEMF groups. ${ }^{*} \mathrm{P}<0.01$ vs. Sham group; ${ }^{\#} \mathrm{P}<0.05$ vs. SCI group. $\mathrm{SCI}$, spinal cord injury; LPEMF, low-frequency pulsed electromagnetic fields; iNOS, inducible nitric oxide synthase.

the microenvironment comprised of neurons, astrocytes, oligodendrocytes, microglial cells and vascular endothelial cells (26). It is well established that the secondary injury is far more important than primary injury, due the crucial role of the microenvironment in tissue preservation and regeneration (7). In the current study, LPEMF treatment improved the recovery of motor function in the SCI rats and alleviated the inflammation and oxidative stress in the damaged spinal cord, which indicated that LPEMFs promote functional recovery, which may be associated with reduced secondary injury following SCI.

Inflammatory cascades are activated during secondary injury following SCI. High levels of pro-inflammatory factors are released from spinal cord tissue (astrocytes and microglia) and peripheral cells (neutrophils, monocytes and macrophages) to increase vascular permeability. TNF- $\alpha$ and IL-1 $\beta$ pathophysiological signaling pathways are two of the most important components in SCI inflammatory cascades. The increased expression of TNF- $\alpha$ and IL-1 $\beta$ can suppress cell survival and lead to cell death. The NF-кB signaling pathway has been well established as the center of the pathophysiology of inflammatory reactions induced by SCI (27). The NF- $\kappa B$ can be elevated by the pro-inflammatory cytokines and chemokines following injury. The activation of NF- $\kappa \mathrm{B}$, as a transcription factor, is required for the upregulation of TNF- $\alpha$ and IL- $1 \beta$. Therefore, this positive feedback amplifies the inflammatory reaction and exacerbated the microenvironment following SCI (28). Thus, the results of the current study suggest that LPEMFs can reduce the expression of TNF- $\alpha$, IL- $1 \beta$ and NF- $\kappa B$ to alleviate the inflammatory reaction induced by SCI, and this supports previous reports demonstrating LPEMF exposure exhibits anti-inflammatory effects in synoviocytes, chondrocytes and

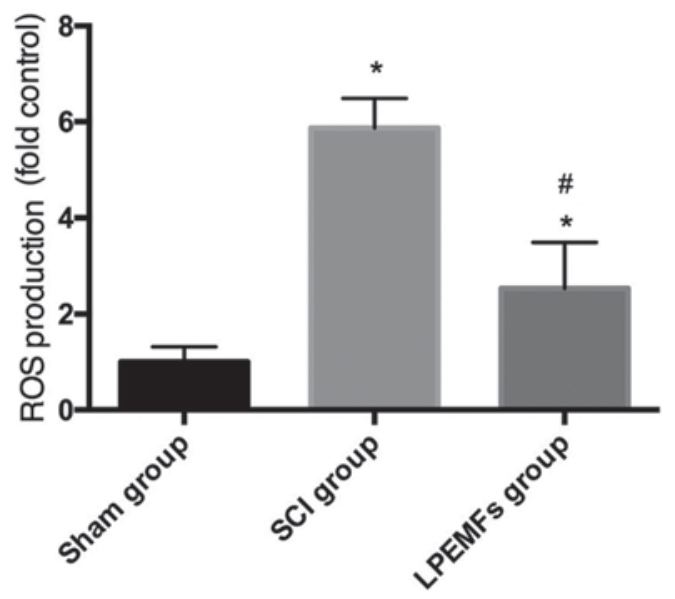

Figure 5. Effect of LPEMFs on the ROS level following SCI. ROS were detected at 14 days post-injury. ROS assay kit was used to determine the production of ROS. "P<0.01 vs. Sham group; ${ }^{*} \mathrm{P}<0.05$ vs. SCI group. ROS, reactive oxygen species; SCI, spinal cord injury; LPEMF, low-frequency pulsed electromagnetic fields.

osteoblasts (29-31). However, in the current study, inflammation was only detected at $14 \mathrm{dpi}$. It will be necessary to use other time points to validate these results.

Following SCI, the production and elimination of oxidative species are imbalanced, which results in tissue oxidative stress (32). ROS and NO are two main end-products of oxidative stress. Superoxides, hydroxylradicals, hydrogen peroxides and peroxynitrites are the principal components of ROS (33). NO is synthesized by NOS, and the most abundant isoform is the iNOS, which is located in a variety of cell types in the spinal cord and can represent the production of NO (34). Antioxidant enzymes, including superoxide dismutase (SOD) and catalase (CAT), act to reduce oxidative stress. However, damage of the spinal cord impairs their protective ability (35). In the current study, the administration of LPEMFs significantly reduced the levels of ROS and iNOS in the injured spinal cord. By contrast, the expression of CAT and SOD were upregulated by LPEMFs, which can protect damaged tissue. A previous study also demonstrated the antioxidant protective effects of LPEMFs in neuronal cell lines by elevating endogenous antioxidant properties (36), which was in accordance with the current study.

Heat shock proteins have an important role in transport and folding proteins, which is necessary for numerous biological processes. HSP70 has been considered to protect against cellular stress. HSP70 is also modulates inflammation and oxidative stress $(37,38)$. In an SCI model, HSP70 was demonstrated to promote the survival of motor neurons (39). In the current study, LPEMFs significantly promoted the expression of HSP70 in the ventral horn of the injured spinal cord, where the motor neurons are located. Therefore, the enhanced tolerance to inflammation and oxidative stress may be mediated by upregulation of HSP70 following LPEMF treatment. However, the causal association between HSP70 and inflammation/oxidative stress requires further investigation.

In conclusion, the findings of the present study revealed that the administration of LPEMFs reduces inflammation and oxidative stress to promote functional recovery following SCI, and the potential mechanism involves the activation of 
A

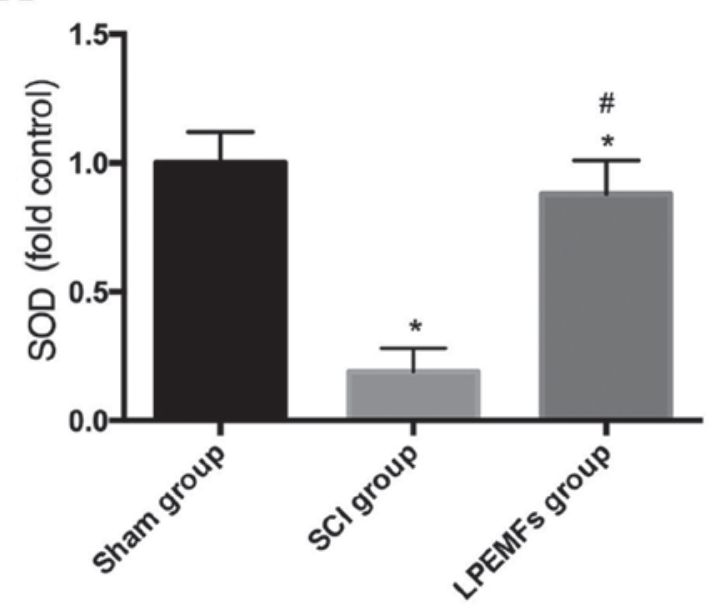

B

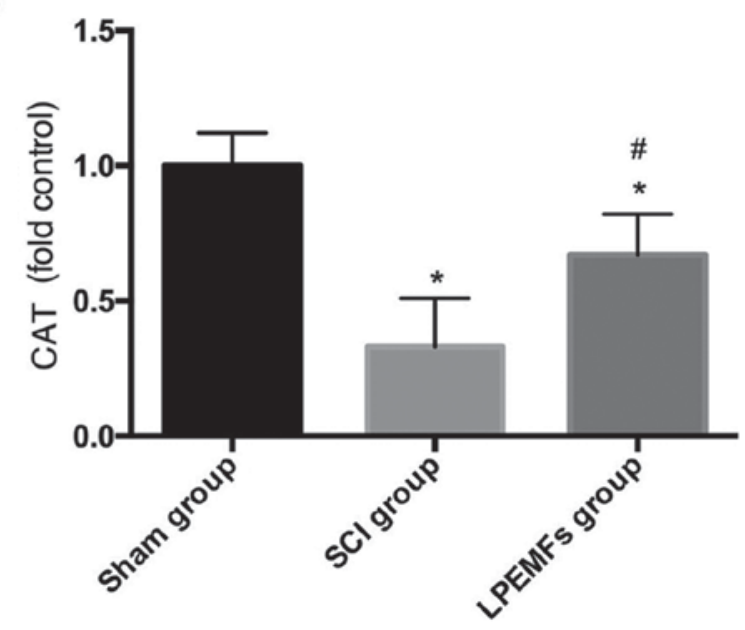

Figure 6. Effect of LPEMFs on the expression of antioxidant enzymes following SCI. SOD and CAT were detected at 14 days post-injury. Expression of (A) SOD and (B) CAT in the spinal cord was measured using ELISA. "P $<0.01$ vs. Sham group; ${ }^{*} \mathrm{P}<0.05$ vs. SCI group. SOD, superoxide dismutase; CAT, catalase; SCI, spinal cord injury; LPEMF, low-frequency pulsed electromagnetic fields.

A
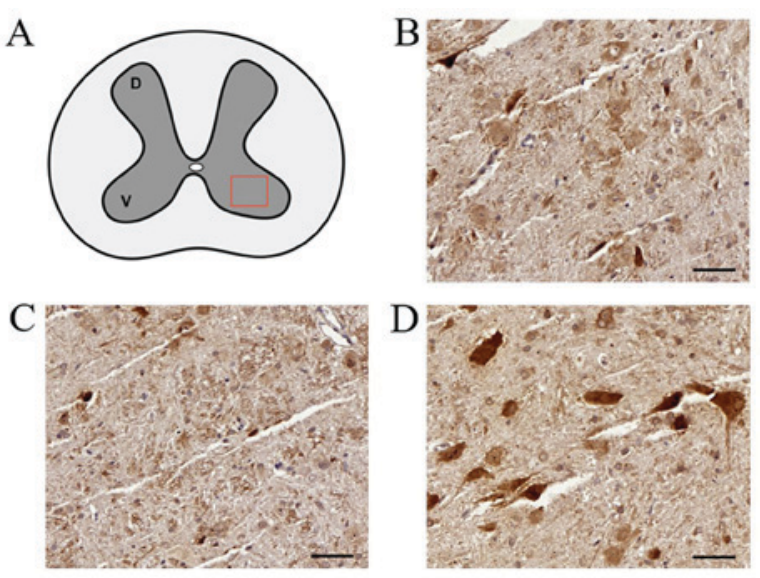

$\mathrm{D}$

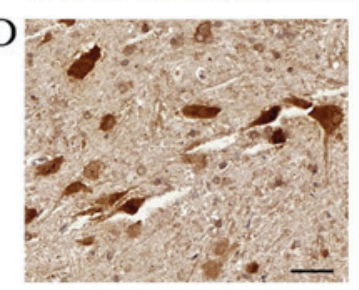

E

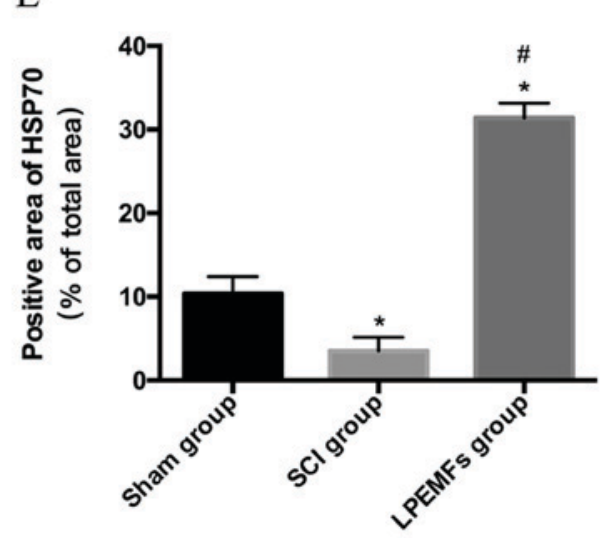

Figure 7. Expression of HSP70 in ventral horn of the spinal cord. HSP70 was detected at 14 days post-injury. (A) Diagram of the spinal cord. Representative image of HSP70 expression in the ventral horn of the spinal cord in the (B) sham group, (C) SCI group and (D) LPEMF group. Scale bar, $50 \mu \mathrm{m}$. (E) Statistical comparison among the three groups. ${ }^{*} \mathrm{P}<0.01$ vs. Sham group; ${ }^{*} \mathrm{P}<0.05$ vs. SCI group. HSP70, heat shock protein 70 ; SCI, spinal cord injury; LPEMF, low-frequency pulsed electromagnetic fields.

HSP70. The findings provide new perspective for identifying novel noninvasive therapeutic methods for early intervention following SCI.

\section{Acknowledgements}

The authors are grateful for the valuable suggestions of Professor Xiaohong Kong from the 221 Laboratory, School of Medicine, Nankai University (Tianjin, China).

\section{Funding}

The authors thank the following sources for funding support: the NSFC program (grant nos. 81330042, 81472070, 81772342 and 81620108018), the Ministry of Science and Technology, China (grant no. 2014DFR31210), and the Tianjin Science and Technology Committee, China (grant nos. 13RCGFSY19000 and 14ZCZDSY00044).

\section{Availability of data and materials}

The data and materials used or analysed during the current study are available from the corresponding author on reasonable request.

\section{Authors' contributions}

SF, CXW, CYW and YL conceived and designed the experiments. CYW and YL performed the experiments. YW, ZW and DS provided critical reagents and scientific input. GN and QW maintained the animals. CYW, YL, SF and CXW analyzed data and prepared the manuscript.

\section{Ethics approval and consent to participate}

All animal experiments were approved by the Animal Welfare Committee of Tianjin Medical University (Tianjin, China), which is based on the NIH Guide for the Care and Use of Laboratory Animals.

\section{Patient consent for publication}

Not applicable. 


\section{Competing interests}

The authors declare that they have no competing interests.

\section{References}

1. Ropper AE and Ropper AH: Acute spinal cord compression. N Eng J Med 376: 1358-1369, 2017.

2. Furlan JC, Sakakibara BM, Miller WC and Krassioukov AV: Global incidence and prevalence of traumatic spinal cord injury. Can J Neurol Sci 40: 456-464, 2013.

3. Jain NB, Ayers GD, Peterson EN, Harris MB, Morse L, O'Connor KC and Garshick E: Traumatic spinal cord injury in the United States, 1993-2012. JAMA 313: 2236-2243, 2015.

4. Miller LE and Herbert WG: Health and economic benefits of physical activity for patients with spinal cord injury. Clinicoecon Outcomes Res 8: 551-558, 2016.

5. Hurlbert RJ,Hadley MN, Walters BC,Aarabi B,Dhall SS, Gelb DE Rozzelle CJ, Ryken TC and Theodore N: Pharmacological therapy for acute spinal cord injury. Neurosurgery 72 (Suppl 2): S93-S105, 2013.

6. Herzer KR, Chen Y, Heinemann AW and González-Fernández M Association between time to rehabilitation and outcomes after traumatic spinal cord injury. Arch Phys Med Rehabil 97: 1620-1627.e4, 2016.

7. Anwar MA, Al Shehabi TS and Eid AH: Inflammogenesis of secondary spinal cord injury. Front Cell Neurosci 10: 98, 2016.

8. Huang JH, Yin XM, Xu Y, Xu CC, Lin X, Ye FB, Cao Y and Lin FY: Systemic administration of exosomes released from mesenchymal stromal cells attenuates apoptosis, inflammation, and promotes angiogenesis after spinal cord injury in rats. J Neurotrauma 34: 3388-3396, 2017.

9. Xun C, Mamat M, Guo H, Mamati P, Sheng J, Zhang J, Xu T, Liang W, Cao R and Sheng W: Tocotrienol alleviates inflammation and oxidative stress in a rat model of spinal cord injury via suppression of transforming growth factor- $\beta$. Exp Ther Med 14: 431-438, 2017.

10. Zou J, Chen Y, Qian J and Yang H: Effect of a low-frequency pulsed electromagnetic field on expression and secretion of IL-1 $\beta$ and TNF- $\alpha$ in nucleus pulposus cells. J Int Med Res 45: 462-470, 2017.

11. Ehnert S, Fentz AK, Schreiner A, Birk J, Wilbrand B, Ziegler P, Reumann MK, Wang H, Falldorf K and Nussler AK: Extremely low frequency pulsed electromagnetic fields cause antioxidative defense mechanisms in human osteoblasts via induction of $\bullet \mathrm{O}_{2}$ and $\mathrm{H}_{2} \mathrm{O}_{2}$. Sci Rep 7: 14544, 2017

12. Urnukhsaikhan E, Mishig-Ochir T, Kim SC, Park JK and Seo YK: Neuroprotective effect of low frequency-pulsed electromagnetic fields in ischemic stroke. Appl Biochem Biotechnol 181: 1360-1371, 2017.

13. Capelli E, Torrisi F, Venturini L, Granato M, Fassina L, Lupo GF and Ricevuti G: Low-frequency pulsed electromagnetic field is able to modulate miRNAs in an experimental cell model of Alzheimer's disease. J Healthc Eng 2017: 2530270, 2017.

14. Dey S, Bose S, Kumar S, Rathore R, Mathur R and Jain S: Extremely low frequency magnetic field protects injured spinal cord from the microglia- and iron-induced tissue damage. Electromagn Biol Med 36: 330-340, 2017.

15. NIH (National Institutes of Health U.S.A): Guide for the Care and Use of Laboratory Animals. The National Academies Press, Washington, DC, pp246, 2011

16. Zhou H, Li X, Wu Q, Li F, Fu Z, Liu C, Liang Z, Chu T, Wang T, Lu L, et al: shRNA against PTEN promotes neurite outgrowth of cortical neurons and functional recovery in spinal cord contusion rats. Regen Med 10: 411-429, 2015.

17. Basso DM, Beattie MS and Bresnahan JC: A sensitive and reliable locomotor rating scale for open field testing in rats. J Neurotrauma 12: 1-21, 1995.

18. Wei ZJ,Zhou XH, Fan BY, Lin W, Ren YM and Feng SQ: Proteomic and bioinformatic analyses of spinal cord injury-induced skeletal muscle atrophy in rats. Mol Med Rep 14: 165-174, 2016.

19. Xu L, Botchway BOA, Zhang S, Zhou J and Liu X: Inhibition of NF- $\mathrm{NB}$ signaling pathway by resveratrol improves spinal cord injury. Front Neurosci 12: 690, 2018.

20. Ferroni L, Tocco I, De Pieri A, Menarin M, Fermi E, Piattelli A, Gardin C and Zavan B: Pulsed magnetic therapy increases osteogenic differentiation of mesenchymal stem cells only if they are pre-committed. Life Sci 152: 44-51, 2016.
21. Ottani V, De Pasquale V, Govoni P, Franchi M, Zaniol P and Ruggeri A: Effects of pulsed extremely-low-frequency magnetic fields on skin wounds in the rat. Bioelectromagnetics 9: 53-62,1988.

22. Jorgensen WA, Frome BM and Wallach C: Electrochemical therapy of pelvic pain: Effects of pulsed electromagnetic fields (PEMF) on tissue trauma. Eur J Surg Suppl: 83-86, 1994.

23. Kavand H, Haghighipour N, Zeynali B, Seyedjafari E and Abdemami B: Extremely low frequency electromagnetic field in mesenchymal stem cells gene regulation: Chondrogenic markers evaluation. Artif Organs 40: 929-937, 2016.

24. Feng SQ, Zhou XF, Rush RA and Ferguson IA: Graft of pre-injured sural nerve promotes regeneration of corticospinal tract and functional recovery in rats with chronic spinal cord injury. Brain Res 1209: 40-48, 2008.

25. Feng SQ, Kong XH, Guo SF, Wang P, Li L, Zhong JH and Zhou XF: Treatment of spinal cord injury with co-grafts of genetically modified Schwann cells and fetal spinal cord cell suspension in the rat. Neurotox Res 7: 169-177, 2005.

26. Kjell $\mathrm{J}$ and Olson L: Rat models of spinal cord injury: From pathology to potential therapies. Dis Model Mech 9: 1125-1137, 2016.

27. Cavalli G and Dinarello CA: Suppression of inflammation and acquired immunity by IL-37. Immunol Rev 281: 179-190, 2018.

28. Ni H, Jin W, Zhu T, Wang J, Yuan B, Jiang J, Liang W and Ma Z: Curcumin modulates TLR4/NF- $x \mathrm{~B}$ inflammatory signaling pathway following traumatic spinal cord injury in rats. J Spinal Cord Med 38: 199-206, 2015.

29. Varani K, De Mattei M, Vincenzi F, Gessi S, Merighi S, Pellati A, Ongaro A, Caruso A, Cadossi R and Borea PA: Characterization of adenosine receptors in bovine chondrocytes and fibroblast-like synoviocytes exposed to low frequency low energy pulsed electromagnetic fields. Osteoarthritis Cartilage 16: 292-304, 2008.

30. Vincenzi F, Targa M, Corciulo C, Gessi S, Merighi S, Setti S, Cadossi R, Goldring MB, Borea PA and Varani K: Pulsed electromagnetic fields increased the anti-inflammatory effect of $\mathrm{A}_{2} \mathrm{~A}$ and $\mathrm{A}_{3}$ adenosine receptors in human T/C-28a2 chondrocytes and hFOB 1.19 osteoblasts. PLoS One 8: e65561, 2013.

31. Ongaro A, Varani K, Masieri FF, Pellati A, Massari L, Cadossi R, Vincenzi F, Borea PA, Fini M, Caruso A and De Mattei M: Electromagnetic fields (EMFs) and adenosine receptors modulate prostaglandin $\mathrm{E}(2)$ and cytokine release in human osteoarthritic synovial fibroblasts. J Cell Physiol 227: 2461-2469, 2012.

32. Visavadiya NP, Patel SP, VanRooyen JL, Sullivan PG and Rabchevsky AG: Cellular and subcellular oxidative stress parameters following severe spinal cord injury. Redox Biol 8: 59-67, 2016

33. Yang Y, Bazhin AV, Werner J and Karakhanova S: Reactive oxygen species in the immune system. Int Rev Immunol 32: 249-270, 2013

34. Sheng W, Zong Y, Mohammad A, Ajit D, Cui J, Han D, Hamilton JL, Simonyi A, Sun AY, Gu Z, et al: Pro-inflammatory cytokines and lipopolysaccharide induce changes in cell morphology, and upregulation of ERK1/2, iNOS and SPLA -IIA $_{2}$ expression in astrocytes and microglia. J Neuroinflammation 8 121, 2011.

35. Wang Q, Chen Q, Ding Q, Yang Q, Peng Y, Lu Y, Deng J and Xiong L: Sevoflurane postconditioning attenuates spinal cord reperfusion injury through free radicals-mediated up-regulation of antioxidant enzymes in rabbits. J Surg Res 169: 292-300, 2011

36. Vincenzi F, Ravani A, Pasquini S, Merighi S, Gessi S, Setti S, Cadossi R, Borea PA and Varani K: Pulsed electromagnetic field exposure reduces Hypoxia and inflammation damage in neuron-like and microglial cells. J Cell Physiol 232: 1200-1208, 2017.

37. Jacquier-Sarlin MR, Fuller K, Dinh-Xuan AT, Richard MJ and Polla BS: Protective effects of hsp70 in inflammation. Experientia 50: 1031-1038, 1994.

38. Sevin M, Girodon F, Garrido C and de Thonel A: HSP90 and HSP70: Implication in inflammation processes and therapeutic approaches for myeloproliferative neoplasms. Mediators Inflamm 2015: 970242, 2015.

39. Shabbir A, Bianchetti E, Cargonja R, Petrovic A, Mladinic M, Pilipović K and Nistri A: Role of HSP70 in motoneuron survival after excitotoxic stress in a rat spinal cord injury model in vitro. Eur J Neurosci 42: 3054-3065, 2015.

This work is licensed under a Creative Commons Attribution-NonCommercial-NoDerivatives 4.0 International (CC BY-NC-ND 4.0) License. 